Recepción: 15/ 11/ 2016

Aceptación: 22 / 02/ 2017

Publicación: 06/ 03/2017
Ciencias de la salud

Artículo de investigación

\title{
Algunas consideraciones acerca de la radiografía panorámica
}

Some considerations about panoramic radiography

Algumas considerações sobre a radiografia panorâmica

\author{
Maholy D. Muñoz Macías I \\ maholymunozm@hotmail.com \\ Nicolle V. Muñoz Macías II \\ nicolle@hotmail.com \\ Julio C. Jimbo Mendoza ${ }^{\text {III }}$ \\ juliojimbomendoza@gmail.com
}

Correspondencia: albamariamc@ hotmail.com

\footnotetext{
I Odontóloga, Docente de la Universidad Laica Eloy Alfaro de Manabí, Manta, Ecuador.

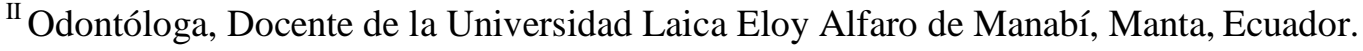

III Magister en Salud Publica Mención Gerencia de los Servicios de Salud, Doctor en Odontología, Diploma Superior en Educación Universitaria por Competencias, Docente de la Universidad Laica Eloy Alfaro de Manabí, Manta, Ecuador.
} 


\title{
Resumen
}

Conocer la importancia y utilidad de las radiografías panorámicas, servirá para tomar conciencia de los avances que ha sufrido el sector de la Odontología. En este sentido, es un examen complementario de inestimable valor en el diagnóstico de lesiones del complejo bucomaxilofacial, y para la conservación en archivo, siendo de gran valor en cuestiones de orden legal. A tales efectos se realizó una revisión bibliográfica exhaustiva para exponer algunos aspectos relacionados con esta temática.

Palabras clave: odontología; rayos X; radiografía panorámica.

\begin{abstract}
Knowing the importance and usefulness of panoramic radiographs will serve to become aware of the advances that have undergone the Dentistry sector. In this sense, it is a complementary examination of inestimable value in the diagnosis of lesions of the complex buco-maxillofacial, and for the conservation in archives, being of great value in questions of legal order. To that end, a comprehensive bibliographic review was carried out to present some aspects related to this topic.
\end{abstract}

Key words: dentistry; $\mathrm{x}$-rays; panoramic radiography.

\section{Resumo}

Sabendo da importância e utilidade das radiografias panorâmicas servirá para aumentar a conscientização sobre o progresso que tem sido no campo da odontologia. A este respeito, uma análise mais aprofundada é inestimável no diagnóstico de lesões maxilo-facial complexo e arquivo de conservação, sendo de grande valor em questões de ordem jurídica. Para este efeito, uma ampla revisão bibliográfica foi conduzida para expor alguns aspectos relacionados a este assunto.

Palavras chave: odontologia; raios-X; radiografia panorâmica.

\section{Introducción}

La evolución histórica de los hallazgos naturales y su relación con los fenómenos y asociaciones científicas durante la antigüedad, han promovido de manera notable, el desarrollo de la ciencia. Es así que la filosofía pragmática del siglo XVII, se convierte en ciencia aplicativa, cuando numerosos investigadores intentan dar razón científica a aquellos hallazgos empíricos, como 
ocurre con la electricidad y el electromagnetismo, observado en fenómenos naturales como los truenos, rayos, etc., así como la atracción de los cuerpos hacia el centro terrestre. ${ }^{1}$

Es de esta forma que los rayos $\mathrm{X}$, llamados así por su descubridor, se convierten en uno de los sucesos más importantes en la historia de la medicina, permitiendo a la comunidad científica, la observación del interior sólido del cuerpo humano. ${ }^{1}$

\section{Antecedentes históricos de la radiografía}

Los rayos $\mathrm{X}$ son el resultado de radiación electromagnética penetrante, con una longitud de onda menor que la luz visible, producida bombardeando un blanco (generalmente de volframio) con electrones de alta velocidad. ${ }^{2}$

Los rayos $\mathrm{X}$ fueron descubiertos de forma accidental en 1895 por el físico alemán Wilhelm Conrad Roentgen mientras estudiaba los rayos catódicos en un tubo de descarga gaseosa de alto voltaje. A pesar de que el tubo estaba dentro de una caja de cartón negro, Roentgen vio que una pantalla de platinocianuro de bario, que casualmente estaba cerca, emitía luz fluorescente siempre que funcionaba el tubo. Tras realizar experimentos adicionales, determinó que la fluorescencia se debía a una radiación invisible más penetrante que la radiación ultravioleta. Roentgen llamó a los rayos invisibles "rayos X" por su naturaleza desconocida. Posteriormente, los rayos X fueron también denominados rayos Roentgen en su honor. ${ }^{2}$

La siguiente gran mejora se llevó a cabo en 1913, por el físico estadounidense William David Coolidge. El tubo de Coolidge tiene un vacío muy alto y contiene un filamento calentado y un blanco. Esencialmente, es un tubo de vacío termoiónico en el que el cátodo emite electrones al ser calentado por una corriente auxiliar, y no al ser golpeado por iones, como ocurría en los anteriores tipos de tubos. Los electrones emitidos por el cátodo calentado se aceleran mediante la aplicación de una alta tensión entre los dos electrodos del tubo. Al aumentar la tensión disminuye la longitud de onda mínima de la radiación. ${ }^{2}$

La mayoría de los tubos de rayos $\mathrm{X}$ que se emplean en la actualidad son tubos de Coolidge modificados. Los tubos más grandes y potentes tienen anticátodos refrigerados por agua para impedir que se fundan por el bombardeo de electrones. El tubo antichoque, muy utilizado actualmente, es una modificación del tubo de Coolidge, con un mejor aislamiento de la carcasa (mediante aceite) y cables de alimentación conectados a tierra. Los aparatos como el betatrón se 
emplean para producir rayos $\mathrm{X}$ muy duros, de longitud de onda menor que la de los rayos gamma emitidos por elementos naturalmente radiactivos. ${ }^{2}$

En el Campo Médico se llegó a utilizar la radiología como medio auxiliar de diagnóstico desde 1896, permitiendo la ubicación de algunas afecciones que presentaban los pacientes. Estos prototipos iníciales, fueron evolucionando tecnológicamente dando lugar a la aparición de la medicina aplicativa contemporánea, donde el uso radiológico se convierte en un instrumento tecnológicamente más activo de observación, ofreciendo mayor seguridad, menor exposición y mayor calidad de imagen, de esta forma, el uso de contrastes, radioisótopos y marcadores, fueron incluyéndose en la imagenología, dando lugar a observaciones anatómicas y funcionales de órganos que antes no podían ser vistos con el uso de la radiografía convencional. La introducción de la radiología digital, amplió el campo imagenológico, permitiendo observar con mayor nitidez y cercanía, estructuras que antes no podían lograr acercamientos, como los observados con estos nuevos instrumentos, los cuales además ofrecían tiempos de exposición y revelado muy bajos y costes económicos menores. ${ }^{3,1}$

Su popularización se extendió a otros campos como la Odontología, donde Frederic Otto Walkhoff, profesor de la Universidad de Braunshweig, en 1896 consiguió tomar una foto a su molar, utilizando un tiempo de exposición a los rayos X de 25 minutos. ${ }^{3}$

\section{La radiografía en Odontología}

El examen radiográfico es un examen complementario de inestimable valor en el diagnóstico de lesiones del complejo buco-maxilofacial. Para que él proporcione la información necesaria es esencial que presente una imagen de calidad, considerando las limitaciones provenientes de la obtención de una imagen bidimensional de una estructura tridimensional. Caso contrario, el diagnóstico puede ser perjudicado. Además de la importancia diagnóstica, la calidad de la imagen radiográfica es fundamental para la conservación en archivo, siendo de gran valor en cuestiones de orden legal. ${ }^{4}$

Se ha estimado que el radiodiagnóstico dental, contribuye de gran manera a la colectiva anual global, con un aporte sobre la población mundial de aproximadamente 14.000 Sv/hombre, que equivale a $0.002 \mathrm{mSv} /$ persona. Aproximadamente, el $68 \%$ de esta cuota colectiva global se englobaría dentro de los países de nivel. ${ }^{4}$ 


\section{Tipos de radiografías dentales}

Las técnicas de imagen son las tecnologías más utilizadas en las clínicas dentales por la importante ayuda que ofrecen en el diagnóstico rápido y eficaz de diversos problemas relacionados con la salud bucodental. ${ }^{5}$

Ante esto hay que tener en cuenta los diferentes tipos de radiografías dentales, puesto que existen diferentes tecnologías y formas de tomar estas imágenes, que suelen ir en función de la parte de la boca que se desea revisar. ${ }^{5}$

Las radiografías están divididas en dos categorías principales: intraorales y extraorales. Una radiografía intraoral es la que se toma dentro de la boca. Una radiografía extraoral es tomada por fuera de la boca.

Es evidente que el odontólogo tiene ante sí toda una variedad de radiografías dentales, que puede ir utilizando en función de las necesidades que le vayan surgiendo o para el diagnóstico que necesite hacer en un momento determinado.

\section{La radiografía panorámica}

La radiografía panorámica es una técnica radiológica, que muestra las estructuras óseas (los maxilares, la articulación de la mandíbula y los dientes) del rostro del paciente en una sola imagen general. Para ello, se vale de una máquina de rayos $\mathrm{X}$ especial, que rota alrededor de la cabeza del paciente. Este tipo de radiografía dental también se le llama ortopantomografía. ${ }^{6}$

Con una radiografía panorámica, es posible obtener una visión detallada y de conjunto del estado de la salud oral de un paciente, especialmente de aquellos aspectos que quedan ocultos en una exploración visual convencional con el pequeño espejo de los odontólogos. ${ }^{6}$

Gracias a esta técnica, que "mapea" la cara del paciente a partir de varias radiografías convencionales superpuestas, es posible plasmar las estructuras tridimensionales del rostro en una sola imagen en $2 \mathrm{D} .^{6}$

La ortopantomografía, se utiliza de forma sistemática en odontología para planear tratamientos de endodoncia, periodoncia, implantología, ortodoncia y cirugía, entre otros. También se realizan radiografías panorámicas para verificar si hay muelas del juicio impactadas o detectar posibles problemas mandibulares. ${ }^{6}$ 


\section{¿Cómo se realiza una radiografía panorámica?}

La radiografía panorámica u ortopantomografía, se realiza con un aparato radiográfico anclado a través de un brazo a una columna vertical. Este dispositivo realiza un escaneado de la boca con un pequeño giro alrededor de la cabeza del paciente. Para hacer la radiografía, el paciente debe estar de pie o sentado y cubierto por un chaleco de plomo que lo protegerá de la radiación. Para colocarse de forma correcta, debe morder una pequeña pieza de mordida oclusal, que servirá para registrar la inclinación del nivel de mordida e indicar al dispositivo la posición en la que debe detenerse. Para evitar posibles movimientos, el aparato radiográfico dispone de un apoyafrentes y dos apoyasienes ajustables. Tras el escaneado, el dispositivo genera una imagen digital.

El procedimiento para este tipo de radiografías es muy rápido. El profesional debe indicar al paciente como colocarse y ajustar los parámetros del dispositivo, luego el escaneado durará apenas diez segundos, siendo el tiempo efectivo de radiación de entre tan sólo 3 y 5 segundos. ${ }^{7}$

Con las radiografías panorámicas actuales la cantidad de radiación que el paciente recibe se reduce gracias a que la imagen que se genera es digital y necesita menos tiempo de exposición a los rayos X que al grabar la imagen sobre una placa radiográfica. ${ }^{7}$

La radiación que el paciente recibe cuando se realiza una radiografía se mide en sieverts (unidad de dosis efectiva). En una ortopantomografía el paciente recibe una dosis efectiva que puede oscilar entre 5,5 y 22 microsieverts, siendo el promedio global que recibe una persona de 2500 microsieverts al año. ${ }^{7}$

\section{¿Qué se puede estudiar con una ortopantomografía?}

Mediante el estudio de la imagen, se puede valorar el estado de los dientes y estructuras anatómicas adyacentes como:

- La presencia de caries dentales.

- La extensión de las caries dentales y si existe contacto con el nervio dental.

- La presencia de procesos infecciosos que se han extendido más allá del diente.

- Presencia de dientes incluidos en el hueso o que aún no han erupcionado.

- Cronología de la erupción de los dientes, en los niños con dentadura temporal y mixta.

- Resorciones radiculares producidas por procesos infecciosos o por efectos traumáticos, al igual que por ortodoncia. 
- Cantidad de estructura ósea remanente existente para implantes dentales.

- Presencia de lesiones óseas patológicas.

- También se pueden valorar las estructuras anatómicas tales como la articulación temporomandibular, los senos maxilares, el tabique nasal y la presencia de asimetrías óseas. ${ }^{8}$

Las principales ventajas en una radiografía panorámica son:

a) Amplia cobertura de los huesos faciales y de los dientes.

b) Baja dosis de radiación para el paciente.

c) Comodidad del examen para el paciente.

d) Puede emplearse en pacientes que no pueden abrir la boca.

e) Escaso tiempo necesario para hacer la proyección panorámica. ${ }^{9}$

En una investigación realizada por Fuentes Puebla L et, la cual consistió en actualizar los conocimientos sobre los efectos biológicos de los rayos-X, por medio de una revisión bibliográfica en las principales bases de datos médicas (Scielo, Pubmed, EBSCO, Hinari) de 27 materiales científicos escritos y electrónicos relacionados con el tema, utilizando los descriptores de búsqueda: rayos-X, radiaciones ionizantes, efectos nocivos en la práctica estomatológica. Se concluyó, que a pesar de que los pacientes no se someten a altas dosis de energía ionizante en los tratamientos odontológicos, su uso inadecuado e irracional puede traer severas consecuencias. ${ }^{10}$

Según lo expuesto en una investigación realizada por Acha Sinche, en la que se utilizó la radiografía panorámica, con la finalidad de determinar hallazgos patológicos previos al tratamiento de ortodoncia, puso de manifiesto la utilidad de este medio como una herramienta para diagnosticar diversas patologías antes de que afecten al paciente, a nivel oral y con ello, prevenir las complicaciones de patologías silenciosas o ignoradas. ${ }^{9}$

En el futuro, la evolución de las exploraciones diagnósticas se prevé que será tan rápida y compleja, que cada profesional se verá obligado a una mayor especialización dentro de su área, pero permitirá hacer un diagnóstico multidisciplinario, que significará la minimización de los errores y favorecerá la realización de los tratamientos. 


\section{Referencias bibliográficas}

1- Dávalos Villca Maybeli Vivian. Historia de la Radiología. Rev. Act. Clin. Med [Citado 12 junio 2016]. Disponible en:

http://www.revistasbolivianas.org.bo/scielo.php?script=sci_arttext\&pid=S2304$37682013001000001 \& \operatorname{lng}=\mathrm{es}$

2- Manual de radiología para técnicos, Stewart C. Bushon, Octava edicion, Ed. Elsevier Mosby [Citado 12 junio 2016]. Disponible en: -http://www.galeon.com/losrayosx/

3- Padilla A. Ruprecht A. Historia de la radiología oral y maxilo facial, 2007. [Citado 12 mayo]. Disponible en: http://www.saber.ula.ve/bitstream/123456789/29891/1/historia.pdf

4 Campoverde Cuenca S E. Evolución de la radiografía intraoral. 2012 [Citado 12 mayo 2017] Universidad de Guayaquil Facultad piloto de odontología Trabajo de graduación Disponible en: repositorio.ug.edu.ec/bitstream/redug/3031/1/371campoverde.pdf

5- Tipos de radiografías dentales. 2015 [Citado 12 junio 2016]. Disponible en: http://www.bladegrup.com/tipos-de-radiografias-dentales/

6- En que consiste la radiografía panorámica 2017[citado 12 junio 2016]. Disponible en: http://www.sanitas.es/sanitas/seguros/es/particulares/biblioteca-de-salud/saluddental/radiografia_panoramica/index.html

7- Radiografía panorámica u ortopantomografía. [Citado 12 junio 2016]. Disponible en: http://www.clinicadentalmares.com/tratamientos/radiografia_panoramica_u_ortopantomograf ia

8- ¿Para qué sirve una ortopantomografía?2015 [citado 12 junio 2016]. Disponible en: http://estudidentalbarcelona.com/para-que-sirve-una-ortopantomografia/

9- Acha Sinche J. Hallazgos Patológicos en radiografías panorámicas previas al tratamiento de Ortodoncia en el Hospital Central FAP. 2014. [citado 12 junio 2016]. Disponible en: cybertesis.unmsm.edu.pe/bitstream/cybertesis/3585/1/Acha_sj.pdf 
10- Fuentes Puebla Leonor, Felipe Torres Sonia, Valencia Fernández Víctor. Efectos biológicos de los Rayo-X en la práctica de Estomatología. Rev haban cienc méd 2015 [citado 12 junio 2016]; 14(3): 337-347. Disponible en: http://scielo.sld.cu/scielo.php?script=sci_arttext\&pid=S1729- 519X2015000300011\&lng=es 